\title{
Estratégia semiautomática de extração de cruzamentos de vias em áreas urbanas usando ortoimagens de alta resolução
}

\author{
Semiautomatic extraction strategy of urban road junctions from high resolution orthoimages \\ DALMAR JOSÉ DOS SANTOS*, DANIEL RODRIGUES DOS SANTOS * \\ * Universidade Federal do Paraná Setor de Ciências da Terra, Programa de Pós-Graduação em Ciências Geodésicas \\ CEP 81531-Curitiba/PR-Brasil \\ dalmar@ufpr.br; danielsantos@ufpr.br
}

\begin{abstract}
Resumo
Neste trabalho é apresentada e avaliada uma estratégia semiautomática para a extração do cruzamento de vias com o uso de ortoimagens de alta resolução espacial em cenas correspondentes a áreas urbanas densas. Na estratégia proposta a extração da malha viária é realizada através da coleta de um conjunto de dados de treinamento de amostras que gera, no software WEKA, a árvore de decisão contendo o conhecimento explícito das classes presentes nas imagens. Sobre a malha viária definida, são aplicados operadores morfológicos e algoritmos que permitem a geração de eixos de vias (esqueletos), detecção de hipóteses de cruzamentos, seleção de pontos de referência e a extração do cruzamento de vias. A estratégia propostas foi testada com dados reais e os resultados obtidos foram analisados, permitindo uma avaliação da estratégia, bem como das situações potencialmente problemáticas. Os resultados apresentados nos experimentos demonstraram a viabilidade da extração de vias em cenas de áreas urbanas densas, formadas por malhas viárias com cruzamentos simples e complexos.
\end{abstract}

Palavras-chave: Cruzamentos de vias; malha viária; WEKA; segmentação de imagem; morfologia matemática; ortoimagem de alta resolução

\begin{abstract}
The aim of this paper is present and asses a strategy for semi-automatic extraction of road junctions from high resolution orthoimages take over urban areas. The proposed strategy is performed by collecting a set of samples data training and generates decision tree containing the explicit knowledge of the object classes, by WEKA software. On the roads defined, are applied morphological operators and algorithms that allow the detection of skeletons, junctions, selection of reference points and the roads junction extraction. The proposed strategy was tested with real data and the results were analyzed, allowing an evaluation of the strategy, as well as potentially problematic situations. The results presented in the experiments demonstrated the feasibility of road extraction in urban area scenes, road networks formed by simple and complex intersections.
\end{abstract}

Key-words: Road junction; road network; WEKA; image segmentation; mathematical morphology; high resolution orthoimage

\section{Introdução}

O mapeamento de vias e malhas viárias é de fundamental importância para o desenvolvimento econômico de qualquer país, uma vez que representam os meios para o transporte convencional de bens e pessoas. Assim qualquer alteração existente no posicionamento das vias e na malha viária, devem ser registradas nos Sistemas de Informação Geográfica (SIG), para que este contenha a realidade local, a fim de permitir o controle do cadastro de equipamentos públicos de abastecimento de água, serviços de esgotos, energia elétrica, coletas de água pluvial, rede telefônica, rede de gás canalizado, além da localização de imóveis públicos e privados, bem como, suas funções de uso. Uma abordagem alternativa para a criação e gestão da digitalização de dados para compor um SIG é a automação ou semiautomação do processo de deteç̧ão e extração da malha viária. Neste caso, o processo manual de digitalização é, em parte, substituído por um sistema de processamento de imagens digitais, tornando a tarefa menos morosa e, eventualmente, mais rentável.

Estratégias de extração automática e semiautomática de vias ou rodovias em imagens têm sido bastante difundidas na comunidade científica, citam-se os trabalhos publicados por: Baumgartner et al. (1996, 1999a e 1999b); 
Steger (1996 e 1997); Gwinner et al. (1999); Hinz et al. (1999); Dal Poz et al. (2000a, 2000b, 2001, 2003, 2005, 2007, 2009, 2010); Gülch (2000); Pateraki (2002 e 2003); Shukla (2002); Wiedemann (2002); Mena (2003); Sarabandi et al. (2004); Zhou (2005); Kumar (2006); Santos (2006); Mayunga (2007); Ishibashi (2008); Hinz (2008); Maktav (2009); e Mirnalinee(2009); entre outros.

De acordo com Dal Poz (2002) dentro do processo de extração da malha viária, a etapa de detecção e extração dos cruzamentos de vias é essencial para a geração da malha viária. As informações sobre os cruzamentos de vias são importantes componentes da malha viária na segurança do tráfego e na aplicação de tecnologias de posicionamento, tais como: rastreamento de veículos; navegação; entretenimento; negócios; segurança; e sistemas baseados em localização (em inglês, Location Based Systems - LBS).

Entretanto, embora importantes, os cruzamentos de vias são raramente abordados em metodologias para extração de rodovias (BARSI, 2002; RAVANBAKHSH, 2007). Os principais trabalhos relacionados à extração automática e semiautomática de cruzamento de vias são: Mayer (1998); Hinz et al. (1999); Teoh (2000); Barsi (2002); Wiedemann (2002); Vale (2003); Zhang (2003); Gautama et al. (2004); Zanin (2004); Gerke (2006); Ravanbakhsh (2007); Hosomura (2008) e Lin (2008).

Atualmente os estudos sobre a extração do cruzamento de vias estão focados, principalmente, no uso de imagens digitais de cenas de área rural onde a paisagem, geralmente, é menos alterada quando comparada as da área urbana. A melhoria da resposta espectral e geométrica dos objetos que formam as imagens digitais, devido ao avanço tecnológico da resolução espacial e radiométrica dos sensores de captura de imagem; as características de largura e reflectância do conjunto de vias que formam a rede de malha viária nas imagens de cenas de área urbana; e a necessidade de estratégias de extração de rede da malha viária, que minimizem a interação humana no processo de atualização de base de dados SIG, motivam o estudo de estratégias de detecção e extração de cruzamentos em ortoimagens de alta resolução de cenas de áreas urbanas densas.

O objetivo deste trabalho é avaliar em condições reais a potencialidade de uma estratégia semiautomática para a extração do cruzamento de vias com o uso de ortoimagens de alta resolução espacial em cenas correspondentes a áreas urbanas densas. Na estratégia proposta os objetos que compõem a cena urbana são classificados, de forma semiautomática, pelo software de domínio público denominado WEKA (em inglês, Waikato Environment for Knowledge Analysis). Com a estratégia de classificação supervisionada, empregada neste trabalho, a malha viária é segmentada e algoritmos e operadores morfológicos são aplicados para detecção de eixos de vias, pixels de bordas, hipóteses de cruzamentos, pontos de referência e extrair o cruzamento de vias.
Foram conduzidos experimentos com dados reais e os resultados obtidos mostraram que o método é promissor e possui potencialidade na extração do cruzamento de vias. 0 desenvolvimento deste trabalho é motivado por ser um tema em aberto, pela escassez de investigações sobre o assunto proposto, e necessidade de promover a extração de cruzamentos de vias em cenas de áreas urbanas densas e a necessidade de apresentar novas alternativas à comunidade. Enfim, pela relevância científica comprovada, devido à importância dispensada pela ISPRS Commmision III - Photogrammetric Computer Vision and Image Analysis, aos objetos de estudo deste trabalho.

\section{Método}

Objetos antrópicos como casas, edifícios, viadutos, veículos, vias, faixas e os objetos naturais como áreas verdes e sombras, presentes em imagens de alta resolução em áreas urbanas; apresentam uma grande diversidade de tonalidade, tamanho, forma, textura, padrão e localização que dificultam a sua interpretação e extração e por isso devem ser identificados e tratados para que o resultado da extração das vias de seus cruzamentos não seja prejudicado. A figura 1 apresenta exemplos dos objetos que podem ser encontrados em uma imagem de alta resolução em área urbana.
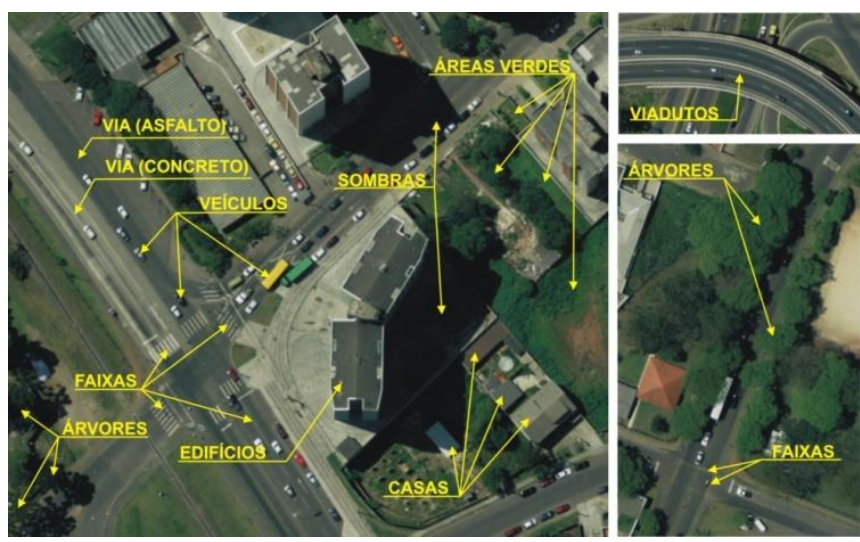

Figura 1 - Objetos presentes em uma porção da ortoimagem RGB em área urbana.

Ao observar o objeto vias, na figura 1, pode ser verificada a complexidade de uma cena urbana em função dos diversos objetos presentes, bem como os casos particulares em que as bordas das vias encontram-se obstruídas por copas de árvores e projeções de sombras. Isto provoca um estreitamento e alteram a quantidade de pixels, classificados como pertencentes ao objeto via. Por isto, estas considerações devem ser levadas em conta durante o processo de classificação dos pixels, para minimizar os seus efeitos para extrair os objetos vias existentes na imagem com melhor eficiência.

A estratégia proposta é dividida em seis etapas: (1) seleção da ortoimagem de alta resolução em uma cena de área urbana densa contendo as vias que formam a rede de malha viária; (2) coletada manual, no software ENVI, do conjunto de dados de treinamento das amostras; (3) 
transformação do arquivo texto do formato ENVI para o formato ARFF; (4) geração da árvore de decisão contendo o conhecimento explícito das classes da imagem a partir da aplicação do arquivo ARFF no WEKA (2009); (5) transformação do arquivo texto com a árvore de decisão para o formato "IF-THEN"; e (6) extração automática dos pontos de referência e do cruzamento das vias. A figura 2 mostra o fluxograma da estratégia semiautomática proposta.

Sobre uma ortoimagem definida na etapa (1), são coletados manualmente os dados do conjunto de treinamento das amostras. Durante a coleta dos dados, na execução da etapa (2), foram gerados, sobre a imagem, pequenos retângulos que contêm a região de pixels das classes da Amostra 01 (área verde, asfalto, calçada, sombra e telhado), e Amostra 02 (área verde, asfalto, calçada, faixas de sinalização, paralelepípedo, solo, sombra e telhado). Ao final da coleta, os arquivos contendo: as descrições das classes, as quantidades de pixels por classes, e os valores RGB de cada pixel coletado; é exportado em um arquivo no formato TXT sendo transformado do formato ENVI para um arquivo no formato ARFF (em inglês, Attribute-Relation File Format) através do Módulo (1) desenvolvido em linguagem $\mathrm{C}++$ Builder (etapa 3 ).

$\mathrm{Na}$ etapa (4), o arquivo texto no formato ARFF é utilizado pelo software WEKA, tendo as seguintes informações, a saber: a declaração da relação que o arquivo representa; uma lista de atributos; a relação das classes que os mesmos podem assumir; o conjunto dos valores dos dados das amostras; e o valor RBG de cada pixel do conjunto de dados de treinamento das amostras.

$O$ conjunto de dados de treinamento (D) é formado por amostras (d1, d2, ..., dk) já classificadas. Cada amostra (d) é um vetor (v1, v2, ...., vk) de valores de atributos ou características da amostra. Alguns testes de limiares ( $T$ ) com resultados mutuamente exclusivos ( $t 1, t 2, \ldots, t k)$ são usados para particionar (D) em subconjuntos (C1, C2, ..., Ck) que representam a classe que pertence a amostra. Estes testes são aplicados recursivamente até a montagem final da árvore de decisão (QUINLAN, 1996).

Desde que não haja casos de valores de atributos idênticos que pertençam a diferentes classes, qualquer teste (T) que produza uma partição não-trivial de (D) conduzirá a um único subconjunto de classes. Para encontrar o limiar ( $t$ ) que maximiza o critério de divisão do conjunto de dados, os valores de atributos em (D) são classificados em ordem distinta (v1, v2, ...., vk). Cada par de valores adjacentes sugerem um potencial limiar (QUINLAN, 1996):

$$
t=\frac{\left(v_{k}+v_{k+1}\right)}{2}
$$

O critério de divisão padrão utilizado pelo algoritmo é a relação de ganho, que representa a diferença entre a quantidade de informação necessária para uma predição correta e as correspondentes quantidades acumuladas dos segmentos resultantes após a introdução de um novo teste para o valor de determinado atributo. Para a avaliação do quanto é oportuno a introdução de um novo teste, são considerados dois momentos: o primeiro, antes da inserção de novos valores dos atributos e o segundo, depois da sua inserção. Se a quantidade de informação requerida é menor depois que a ramificação é introduzida, isto indica que a inclusão deste teste reduz a entropia (desordem) do segmento original. A incerteza do resultado sobre a classe que pertence a proporção de casos em (D) é dada por (QUINLAN, 1996):

$$
\operatorname{Info}(D)=-\sum_{j=1}^{C} p(D, j) * \log _{2}(p(D, j))
$$

Onde, c é o número de classes e $p(D, j)$ a proporção de casos em (D) que pertence a j classes. As informações correspondentes de ganho para o teste (T) com k resultados é dada por (QUINLAN, 1996):

$$
\operatorname{Gain}(D, T)=\operatorname{Info}(D)-\sum_{i=1}^{k} \frac{\left|D_{i}\right|}{|D|} * \operatorname{Info}\left(D_{i}\right)
$$

As informações de ganho obtidas com o teste são fortemente afetadas pelo número de resultados e é máxima quando há um caso em cada subconjunto (Di). Por outro lado, a informação do potencial obtido no particionamento de um conjunto é baseada no conhecimento dos casos de falhas em (Di) que particiona a informação e tendem aumentar com o número de resultados dos testes (QUINLAN, 1996):

$$
\operatorname{Split}(D, T)=-\sum_{i=1}^{k} \frac{\left|D_{i}\right|}{|D|} * \log _{2}\left(\frac{\left|D_{i}\right|}{|D|}\right)
$$

O critério taxa de ganho expressa a proporção de informação gerada pela ramificação e é útil para o processo de classificação. O critério taxa de ganho seleciona um teste que maximize o seu próprio valor, sujeito à restrição que o teste escolhido tenha um ganho de informação pelo menos maior que a média do ganho de informação sobre todos os testes avaliados.

O algoritmo $\mathrm{J48}$ é considerado o algoritmo supervisionado que apresenta o melhor resultado para aquisição do conhecimento e montagem da árvore de decisão. Ele classifica, a partir do conhecimento adquirido, um conjunto de dados desconhecidos sem a necessidade de testar todos os valores dos seus atributos (WITTEN, 2005). Os algoritmos de árvore de decisão começam com um conjunto de dados de treinamento (amostras) e criam uma estrutura em forma de árvore contendo as regras com os atributos que definem as classes. As regras utilizadas em árvores de decisão utilizam a abordagem de "dividir-paraconquistar" onde um problema complexo é decomposto em subproblemas mais simples aplicando recursivamente a 


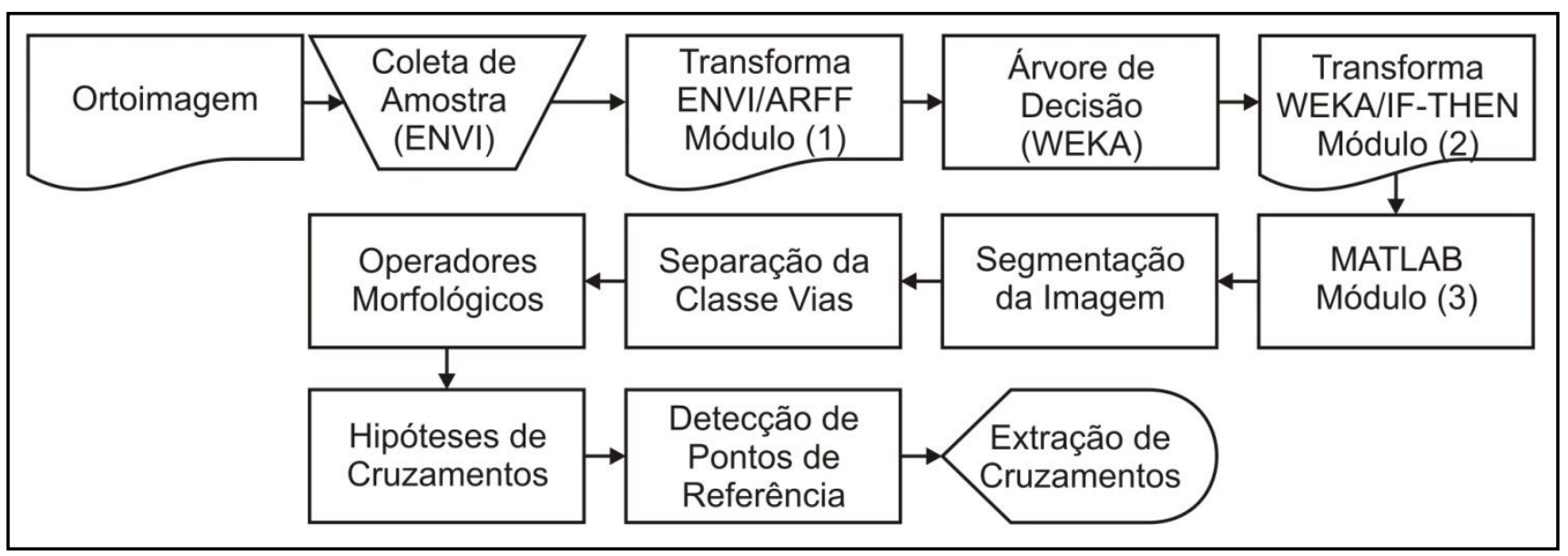

Figura 2 - Fluxograma da estratégia semiautomática proposta.

mesma estratégia a cada subproblema dividindo o espaço definido pelos atributos em subespaços, associando-se a eles uma classe (WITTEN, 2005).

Na etapa (6), o Módulo (3) é responsável pela detecção dos pontos de referência e a extração dos cruzamentos de vias. Neste módulo são realizadas as seguintes tarefas, a saber:

(a) Segmentação da imagem - As classes existentes no conjunto de dados de treinamento geram uma lista ordenada de classes com valores no intervalo de (1 a n). Sobre os pixels da ortoimagem é aplicada a árvore de decisão com o conhecimento explícito das classes existentes no conjunto de dados de treinamento atribuindo aos os pixels o valor definido para a classe;

(b) Separação da classe vias - Das classes obtidas na segmentação, através de uma função expressão condicional do tipo: se<condição>então<conseqüência, a classe vias é separada das demais formando uma imagem binária da classe. Sobre esta imagem é aplicado um filtro de mediana (ver Gonzalez, 2008) que reduz em $30 \%$ a quantidade de pixels do exterior e complementa cerca de $5 \%$ dos pixels no interior das vias;

(c) Aplicação dos operadores morfológicos (ver Gonzalez, 2008) - Sobre a imagem binária resultante de (b) é aplicado um operador morfológico de erosão com um elemento estruturante em forma de disco (com dimensão de $15 \times 15$ pixels) que reduz em $90 \%$ os pixels no exterior e complementa $90 \%$ dos pixels do interior das vias. Para eliminar completamente os pixels do exterior das vias é realizada, pelo algoritmo, uma análise de pertencimento ou não destes pixels ao interior da via. O mesmo caso ocorre no caso de preenchimento dos pixels pertencentes ao interior das vias. Este procedimento elimina 100\% dos pixels classificados erroneamente no exterior da via e preenche $100 \%$ das oclusões causadas por veículos e faixas de sinalização do interior da via. Com isto é formada uma imagem binária contendo somente pixels da pista de rolamento das vias. Sobre a imagem resultante é aplicado um operador morfológico de esqueletização e poda obtendo-se um eixo de via que passa pelo centro da via e permite a aplicação do próximo algoritmo;

(d) Algoritmo de detecção de hipótese de cruzamentos - A partir das bordas da classe vias e dos esqueletos das vias o algoritmo de hipóteses de cruzamentos utilizando uma máscara com dimensão de $3 \times 3$ pixels varre a imagem do esqueleto na busca de pontos pertencentes ao mesmo. Ao encontrar um ponto, captura todos os valores de pixels da imagem do esqueleto, aplicando em seguida um somatório destes valores. Para que um pixel do esqueleto obtenha o status de pertencente à hipótese de cruzamento ele deve atender o critério de que o somatório dos pixels, no interior da máscara, esteja no intervalo entre (3 e 4). Caso o critério seja atendido, a posição que os pixels, com valor unitário, ocupam na mascara $3 \times 3$, determinam o tipo de cruzamento existente. A Tabela 1 apresenta os tipos de cruzamentos considerados nos experimentos. A cor cinza indica a posição ocupada pelo pixel com valor 1 (um) na máscara $3 \times 3$. Caso a distribuição dos pixels unitários da máscara $3 \times 3$ atendam a um dos tipos apresentados na tabela 03 , as coordenadas (linha, coluna) do pixel central da máscara são armazenadas na matriz (Hipóteses de Cruzamentos);

(e) Determinação automática de pontos de referência dos cruzamentos - Para a eliminação dos falsos positivos, a matriz (Hipóteses de Cruzamento) é percorrida do primeiro ao último pixel seguindo os seguintes passos, a saber: (e.1) identificam-se as coordenadas da hipótese de cruzamento utilizando-a como pixel central da aplicação; (e.2) a partir do pixel central define-se um quadrado calculando-se as coordenadas do canto superior esquerdo e canto inferior direito, utilizando o valor 100 pixels; (e.3) sobre as bordas deste quadrado são verificadas os seguintes critérios “(e.3.1) a existência de no mínimo 2 (dois) pixels pertencentes a borda das vias; (e.3.2) a existência de pelo menos dois pixels pertencentes ao esqueleto das vias"; (e.4) caso os critérios (e.3.1) e (e.3.2) sejam atendidos, o status do pixel passa de hipótese de cruzamento para o status ponto de referência dando origem a matriz 
(Cruzamentos) que contem as coordenadas (linha, coluna) dos cruzamentos finais;

(f) Extração do cruzamento de vias - A matriz (Cruzamentos) é percorrida do primeiro ao último pixel seguindo os seguintes passos, a saber: (f.1) identificam-se as coordenadas do pixel do ponto de referência do cruzamento utilizando-o como pixel central da aplicação; (f.2) a partir do pixel central define-se um quadrado calculando-se as coordenadas do canto superior esquerdo e canto inferior direito, utilizando o valor 100 pixels; (f.3) Os pixels das bordas da classe vias são percorridos sendo marcados, como pertencentes aos cruzamentos de vias, aqueles que são pertencentes ao limite interno do quadrado formado em (f.2).

Tabela 1 - Tipos de Cruzamentos

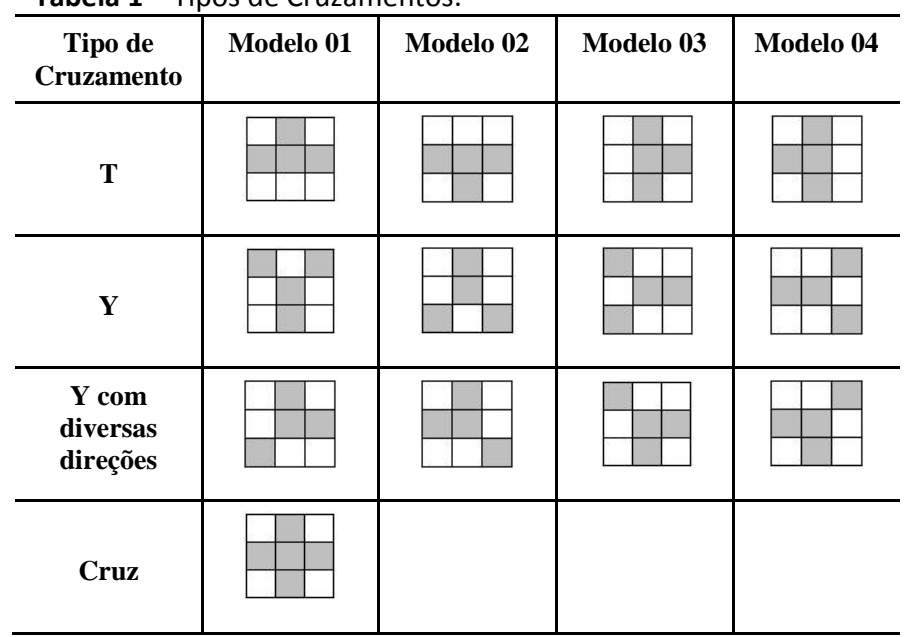

\section{Experimentos e análise dos resultados}

$\mathrm{Na}$ realização dos experimentos foi utilizada uma ortoimagem nas bandas RGB (Red, Green, Blue) de uma cena da área urbana localizada nas proximidades do Campus do Centro Politécnico da Universidade Federal do Paraná - UFPR, em Curitiba, PR. A ortoimagem com resolução espacial de $0,20 \mathrm{~m}$, foi gerada a partir das imagens adquiridas pelo sensor aerotransportado ADS40 e um Modelo Digital do Terreno (MDT) com precisão de $0,5 \mathrm{~m}$. Esta ortoimagem cobre uma área de aproximadamente $1,93 \mathrm{~km} 2$ e tem dimensão de $8.889 \mathrm{x}$ 6.033 pixels, composta por $80 \%$ de objetos antrópicos (feitos pelo homem) e $20 \%$ de objetos naturais. Desta ortoimagem foram retirados 13 (treze) cruzamentos para serem utilizados nos experimentos deste trabalho. A Figura 3 mostra, na cor branca, a localização dos 13 cruzamentos definidos nos experimentos.

A coleta do conjunto de dados de treinamento das amostras é realizada diretamente sobre a ortoimagem e o resultado das árvores de decisão, contendo os conhecimentos das classes, é aplicada diretamente sobre as imagens dos 13 cruzamentos identificados. As ortoimagens de alta resolução, contendo os detalhes e os resultados da localização dos cruzamentos das vias, na aplicação dos conjuntos de dados das amostras 01 e 02 estão demonstradas na tabela 2. Estas imagens têm dimensão de $360 \times 360$ pixels. Para extrair os cruzamentos de vias foi aplicada a seguinte sequência nos experimentos, a saber: segmentação da imagem; separação da classe vias; operadores morfológicos; hipóteses de cruzamento; detecção de pontos de referências dos cruzamentos; e extração de cruzamentos. A tabela 2 apresenta os resultados obtidos na extração de cruzamentos de vias em imagens de alta resolução em cenas de área urbana, onde são apresentados: o número do experimento e as imagens com a indicação da localização do cruzamento encontrado pela aplicação. Abaixo de cada imagem o percentual de acerto do experimento.

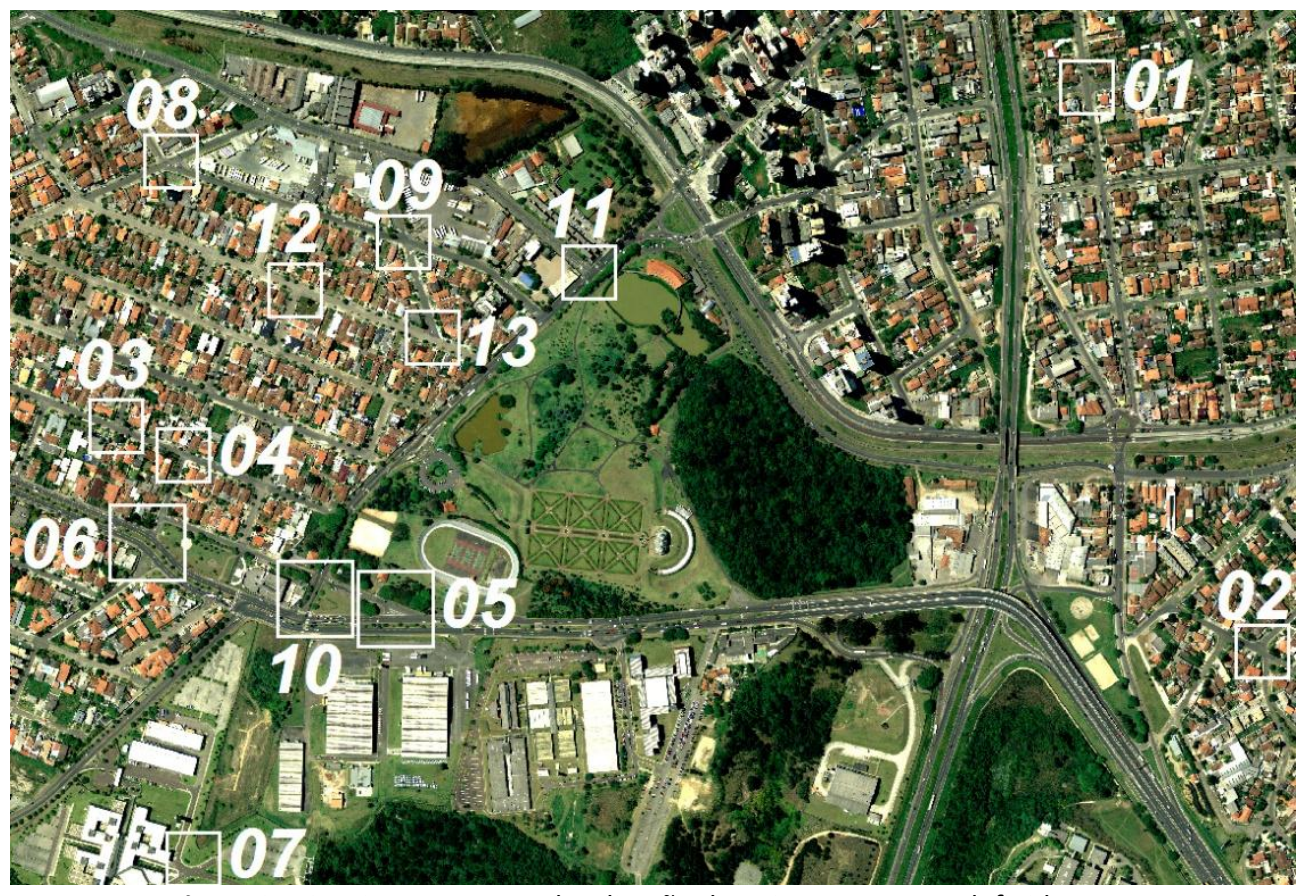

Figura 3 - Ortoimagem com a localização dos 13 cruzamentos definidos. 
Tabela 2 - Resultados obtidos com a aplicação da estratégia proposta.

\begin{tabular}{|c|c|c|}
\hline Experimentos. & $\begin{array}{c}\text { APLIC [01] } \\
\text { Amostra 01 (RGB) } \\
\end{array}$ & $\begin{array}{c}\text { APLIC [02] } \\
\text { Amostra 02 (RGB) } \\
\end{array}$ \\
\hline \multicolumn{3}{|l|}{01} \\
\hline \multicolumn{3}{|l|}{02} \\
\hline \multicolumn{3}{|l|}{$\mathbf{0 3}$} \\
\hline \multicolumn{3}{|l|}{04} \\
\hline \multicolumn{3}{|l|}{05} \\
\hline & (100\% de acerto) & (100\% de acerto) \\
\hline \multicolumn{3}{|l|}{06} \\
\hline & $(80 \%$ de acerto) & (100\% de acerto) \\
\hline 07 & & \\
\hline
\end{tabular}

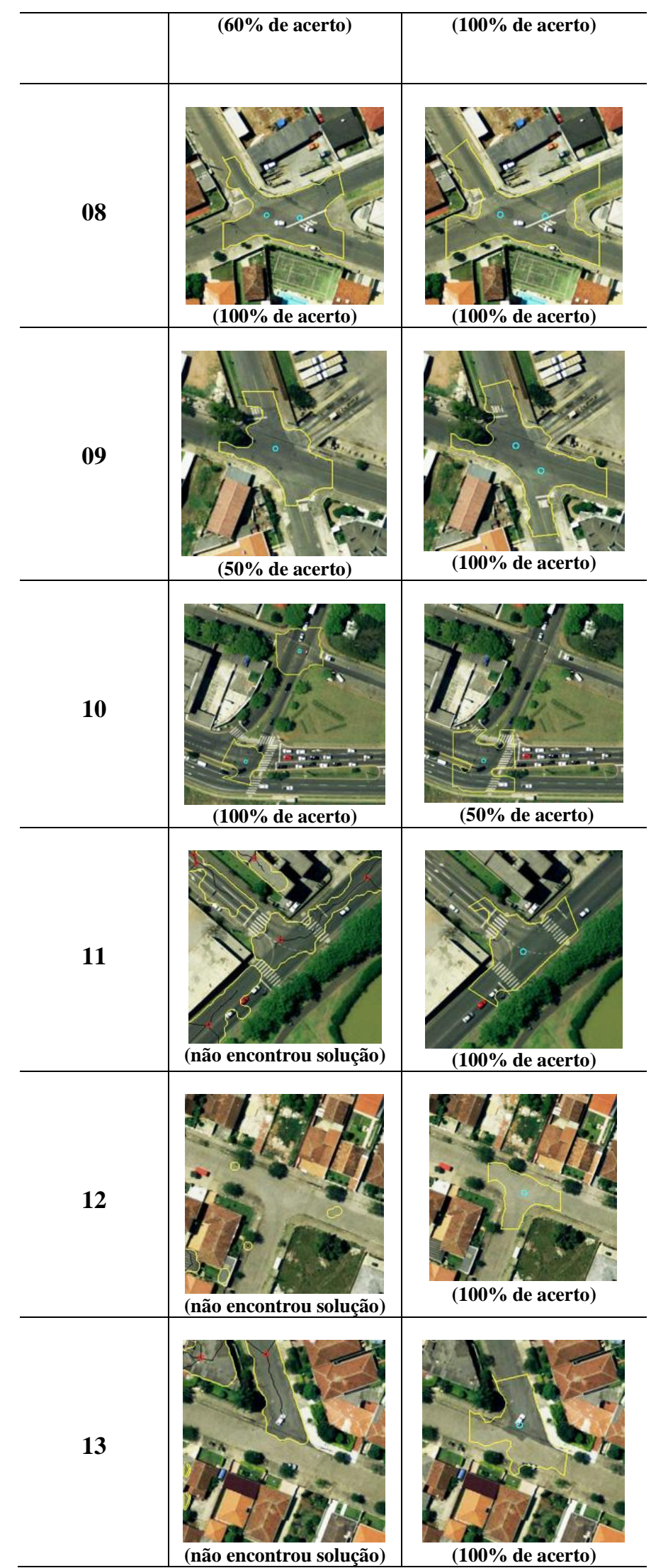

Na tabela 2 as bordas das vias estão representadas na cor amarela, os pontos de referência detectados estão representados na cor ciano, as hipóteses de cruzamentos na cor vermelha e o eixo das vias na cor preta. 
Para auxiliar a avaliação e discussão dos resultados, os cruzamentos foram separados em duas classes, a saber:

- Cruzamentos simples: formados por um único ponto de cruzamento de via do tipo "Cruz (+)" formado por 4 ramos de vias (ver tabela 1 , experimentos 01, 03, 04, 05, 11, 12 e 13);

- Cruzamentos complexos: formados por mais de um ponto de cruzamento de via dos tipos " $\mathrm{T}$ " e " $\mathrm{Y}$ ". Isto permite analisar o comportamento do algoritmo na identificação do cruzamento (ver tabela 1 , experimentos 02, 06, 07, 08, 09 e 10).

A tabela 3 apresenta o resumo geral dos resultados alcançados pela estratégia proposta neste trabalho, entre parênteses estão indicados o número do experimento.

Tabela 3 - Resumo geral dos resultados alcançados pelas estratégias propostas.

\begin{tabular}{|c|c|c|}
\hline Descrição & $\begin{array}{l}\text { APLIC [01] } \\
\text { (RGB) }\end{array}$ & $\begin{array}{l}\text { APLIC [02] } \\
\text { (RGB) }\end{array}$ \\
\hline $\begin{array}{l}\text { (a) Número total de } \\
\text { experimentos. }\end{array}$ & 13 & 13 \\
\hline $\begin{array}{l}\text { (b) Total de experimentos } \\
\text { com } 100 \% \text { de acerto. }\end{array}$ & $\begin{array}{c}7 \\
(01,02,03,04 \\
05,08,10)\end{array}$ & $\begin{array}{c}12 \\
(01,02,03,04 \\
05,06,07,08 \\
09,11,12,13) \\
\end{array}$ \\
\hline $\begin{array}{l}\text { (c) Total de experimentos } \\
\text { com acerto entre } 50 \% \\
\text { e }<100 \% \text {. }\end{array}$ & $\begin{array}{c}3 \\
(06,07,09)\end{array}$ & $\begin{array}{c}1 \\
(10)\end{array}$ \\
\hline $\begin{array}{l}\text { (d) Total de experimentos } \\
\text { resolvidos com } \\
\text { problema. }\end{array}$ & 0 & 0 \\
\hline $\begin{array}{l}\text { (e) Total de experimentos } \\
\text { onde a aplicação não } \\
\text { conseguiu detectar os } \\
\text { pontos de referência } \\
\text { de nos cruzamentos } \\
\text { de vias. }\end{array}$ & $\begin{array}{c}3 \\
(11,12,13)\end{array}$ & 0 \\
\hline $\begin{array}{l}\text { (f) Total de experimentos } \\
\text { com mais de } 50 \% \text { de } \\
\text { solução. }\end{array}$ & 10 & 13 \\
\hline $\begin{array}{l}\text { (g) Percentual, com mais } \\
\text { de } 50 \% \text { de acerto, na } \\
\text { solução da estratégia } \\
\text { proposta para os } \\
\text { experimentos } \\
\text { realizados. }\end{array}$ & $\begin{array}{c}76,92 \% \\
(10 \\
\text { experimentos })\end{array}$ & $\begin{array}{c}100,00 \% \\
(13 \\
\text { experimentos })\end{array}$ \\
\hline $\begin{array}{l}\text { (h) Percentual, com mais } \\
\text { de } 100 \% \text { de acerto, na } \\
\text { solução da estratégia } \\
\text { proposta para a } \\
\text { detecção de pontos de } \\
\text { referência e extração } \\
\text { dos cruzamentos de } \\
\text { vias. }\end{array}$ & $\begin{array}{c}53,85 \% \\
(7 \\
\text { experimentos) }\end{array}$ & $\begin{array}{c}92,30 \% \\
(12 \\
\text { experimentos })\end{array}$ \\
\hline
\end{tabular}

Uma análise visual e estatística das tabelas 2 e 3 mostram que, para o experimento APLIC [01] - realizado com o conjunto de dados de treinamento da Amostra 01, para segmentar os pixels da imagem nas classes "área verde, asfalto, calçada, sombra e telhado" -, considerando que são válidos os experimentos que obtiveram mais do que $50 \%$ dos pontos de referência de cruzamento de vias detectados; de um total de 13 (treze) experimentos, os cruzamentos de vias foram detectados em 10 (dez) deles, resultando um total de $76,92 \%$ de experimentos solucionados sendo que em 3 (três) deles, experimentos (06, 07 e 09) a solução da extração de cruzamentos foi parcial. Em 3 (três) experimentos (11, 12 e 13), não foram encontradas as soluções. Dentre os experimentos realizados com $100 \%$ de acerto na determinação dos pontos de referência e na extração de cruzamentos de vias, são considerados como cruzamentos simples, os experimentos 01, 03, 04, e 05 (ver tabela 2) e cruzamentos complexos, os experimentos 02, 08 e 10 (ver tabela 2).

O resultado da solução parcial do experimento supracitado se deve ao fato que não houve localização de um dos pontos de referência do cruzamento em função da insuficiência de valores espectrais no conjunto de dados de treinamento da Amostra 01, para a obtenção do conhecimento explícito da classe faixa de sinalização. Enquanto os problemas de extração com os demais cruzamentos ocorreram em função da oclusão dos pixels da classe vias ocasionado por veículos, faixas, árvores e sombras sobre a pista de rolamento das vias.

Nos casos onde não foi encontrada solução para a extração dos cruzamentos observa-se que: os cruzamentos são complexos e o objeto da classe faixas de sinalização, não foi considerado no conjunto de dados de treinamento da Amostra 01, reduzindo a eficiência da estratégia proposta em separar os pixels da classe vias. O tipo de pavimentação paralelepípedo (tabela 2; exp. 12 e 13) que reveste a via não foi considerado no conjunto de dados de treinamento da Amostra 01, refletindo na solução do problema, pois o algoritmo não separou os pixels da classe vias.

Para o experimento APLIC [02] - realizado com o conjunto de dados de treinamento da Amostra 02, para segmentar os pixels da imagem nas classes "área verde, asfalto, calçada, faixa de sinalização, paralelepípedo, solo, sombra e telhado" -, foram detectados com $100 \%$ de acerto a quantidade de pontos de referência e a extração do cruzamento de vias em 12 (doze) experimentos, resultando um total de $92,30 \%$ de experimentos solucionados. Para 1 (um) experimento (10) foi encontrada solução parcial na extração dos cruzamentos de vias.

Com o uso da amostra 02, a extração do cruzamento de vias foi solucionada para os experimentos $(11,12$ e 13) onde existe a presença de pixels das classes faixa de sinalização e de pavimentação do tipo paralelepípedo. Esta solução foi possível em função da adição das classes: paralelepípedo e faixas; no conjunto de dados de treinamento da Amostra 02. Com isto o percentual de solução da estratégia semiautomática, para os casos com mais de $50 \%$ de acerto dos pontos de referência de cruzamento de vias detectados, passou de 76,92\% para $100 \%$. Isto só foi possível em função da melhoria no processo de segmentação da imagem e separação dos 
pixels da classe vias. Contudo, o experimento APLIC [02] resolveu parcialmente os pontos de referência e a extração de cruzamentos de vias no experimento (10), devido à oclusão dos pixels da classe vias ocasionado por veículos, faixas e sombras sobre a pista de rolamento das vias.

Também foram aplicados os experimentos APLIC [01] e APLIC [02] sobre uma imagem contendo uma malha viária formada por um total de 10 (dez) cruzamentos de vias. A figura 4 mostra os resultados encontrados.

De um total de 10 (dez) cruzamentos de vias apresentados na figura 4, 7 (sete) cruzamentos obtiveram a sua localização identificada pelas aplicações APLIC [01] e APLIC [02], garantindo um percentual de acerto de $70 \%$ na extração dos cruzamentos de vias. Os problemas de extração com os demais cruzamentos ocorrem em função da oclusão dos pixels da classe vias ocasionado por veículos, faixas, árvores e sombras sobre a pista de rolamento das vias.

Em geral, os casos onde não foram resolvidos os cruzamentos, dizem respeito aos experimentos onde a localização do ponto de referência do cruzamento de via não foi detectada. Isto ocorre por que o critério da não existência de no mínimo 2 (dois) pixels pertencentes à borda do polígono de vias e o critério da existência de pelo menos dois pixels pertencentes ao esqueleto da via não foram atendidos. Esta falha também pode ocorrer se o conjunto de dados de treinamento das amostras, não possuir valores espectrais suficientes que permitiam um conhecimento explícito dos pixels do objeto vias. Isto é fundamental para o correto funcionamento da aplicação das estratégias propostas.

\section{Conclusões}

Neste trabalho foi apresentada uma estratégia semiautomática para detecção e extração do cruzamento de vias com o uso de ortoimagem de alta resolução espacial em cenas correspondentes a áreas urbanas densas. $\mathrm{Na}$ estratégia proposta, os pixels da classe vias são classificados com uso do software WEKA, que aplica árvore de decisão contendo o conhecimento explícito das classes presentes na ortoimagem. Após a classificação dos objetos a geração de eixos de vias, detecção de hipóteses de cruzamentos (pontos de referência) e a extração do cruzamento são realizadas por meio de operadores morfológicos de erosão, dilatação e esqueletização.

A estratégia proposta foi testada com dados reais e os resultados obtidos foram analisados, permitindo uma avaliação global da estratégia, bem como das situações potencialmente problemáticas. Os procedimentos aplicados permitiram o conhecimento explícito: da segmentação da imagem em classes de objetos que a formam; da separação da classe do objeto via; da aplicação da morfologia matemática para solução de oclusões (no interior e nas bordas da via) e a extração do eixo da via; e da detecção e extração dos cruzamentos das vias. Os resultados obtidos mostraram a viabilidade da estratégia proposta para extração de cruzamentos de vias em cenas de áreas urbanas densas, formadas por malhas viárias com cruzamentos simples e complexos.

Foi observado que as oclusões ocasionadas por veículos e sombras de objetos projetadas sobre as bordas das vias, prejudicaram a localização do eixo e, consequentemente, a extração do cruzamento de vias. A coleta das amostras que formam o conjunto de dados de treinamento não foi suficiente para permitir que a árvore de decisão obtivesse um conhecimento explícito para a segmentação das classes presentes nas imagens. Para minimizar este efeito é necessário aumentar a quantidade de pixels coletados em cada amostra, bem como escolher locais nas imagens que apresentem maior diferença na respostas espectrais dos objetos presentes.

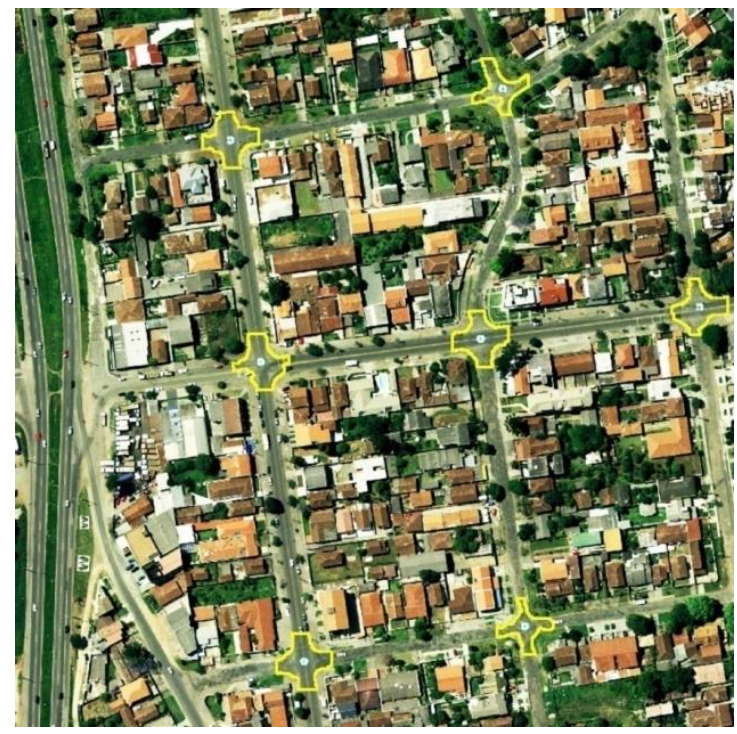

(a)

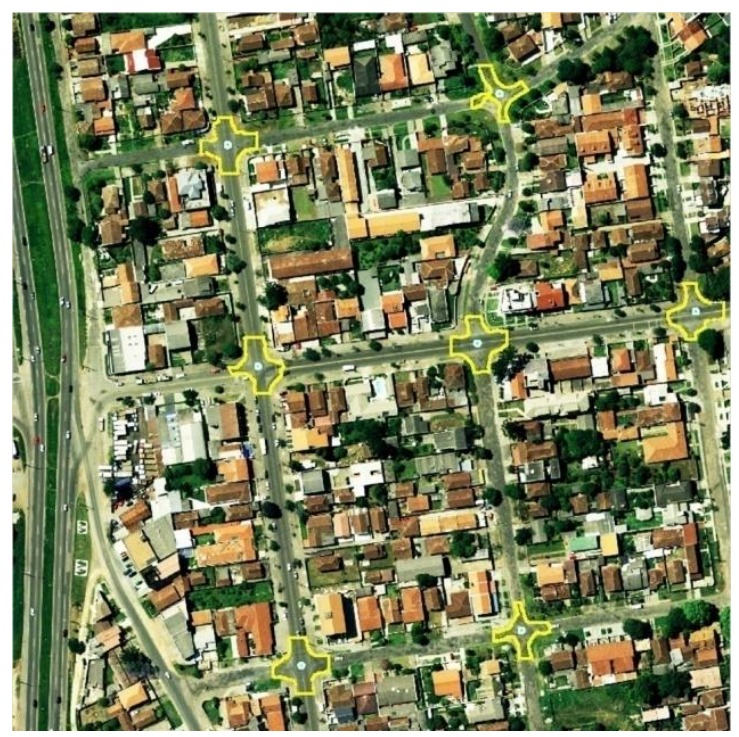

(b)

Figura 4 - Resultados dos cruzamentos de vias em uma malha viária urbana. (a) APLIC [01]; (b) APLIC [02]. 
A aplicação da morfologia matemática reduziu em até $90 \%$ os efeitos dos pixels classificados inadequadamente e nos casos de oclusões provocadas por veículos e faixas localizados no centro das pistas de rolamento das vias, a morfologia matemática aliada à aplicação de fechamento de polígonos resolveram $100 \%$ dos casos. Considerando que são válidos os experimentos que obtiveram mais do que $50 \%$ dos pontos de referência e a extração de cruzamento de vias pode-se afirmar que as estratégias propostas solucionaram $94,44 \%$ dos experimentos. Isto demonstra a viabilidade da estratégia semiautomática proposta para aplicação em ortoimagens RGB.

Como consideração final sobre as estratégias propostas, tem-se que a utilização de técnicas morfológicas aliadas à definição de polígonos da classe vias apresenta grande potencial de detecção de eixos e cruzamentos de vias em imagem digitais de alta resolução respondendo as expectativas esperadas. O tempo de processo dos experimentos variou de acordo com o tamanho de linha e coluna da imagem do cruzamento de via. O uso do MATLAB não se mostrou favorável as aplicações com imagens de dimensão maiores que 1000 x 1000 pixels e esta limitação deve ser considera em futuros desenvolvimentos.

As desvantagens da estratégia proposta em relação às estratégias propostas por: Mayer (1998); Hinz et al. (1999); Teoh (2000); Barsi (2002); Wiedmann (2002); Zhang (2003); Gautama et al. (2004); Zanin (2004); Gerke (2006); Ravanbakhsh (2007); Hosomura (2008) e Lin (2008) são: Como vantagem, tem-se o uso do software WEKA, os conceitos de aprendizagem de máquina, árvore de decisão e a extração do conhecimento explícito de classes de objetos presentes na imagem. Isto garante uma classificação da imagem mais eficiente quando comparada aos métodos de Distância Mínima ou Máxima
Verosssimilhança; e a detecção dos pontos de referência e extração dos cruzamentos das vias em cenas correspondentes a áreas urbanas densas. Como desvantagem tem-se que a imagem RGB é analisada separadamente sem nenhum tipo de fusão de dados; a falta de análise topológica do contexto global detalhando a relação existente entre os objetos que formam a imagem e o material que os compõem no mundo real; A falta de análise topológica de contexto local detalhando a relação entre os veículos e projeções de sombra que prejudicam a definição das vias e em conseqüência a determinação do seu cruzamento; a não identificação e reconstrução das regiões da imagem ocupadas por veículos estacionados nas bordas das vias; e a falta de uma análise mais completa da malha viária existente em imagens onde há vários cruzamentos.

Por fim, recomenda-se a implementação de algoritmos de detecção e análise de áreas de sombra e a localização de faixas e veículos existentes sobre a pista rolamento das vias, para auxiliar a coleta de amostras com a automação da detecção de vias contendo faixas de sinalização reduzindo o trabalho de coleta de amostras. A obtenção de uma largura média da pista de rolamento coletado diretamente sobre os eixos e bordas das vias reduzindo, na classe vias, a influências das obstruções existentes nas bordas das pistas de rolamentos permitindo uma reconstrução mais precisa das vias.

Agradecimentos: Os autores externam seus agradecimentos ao CNPq (Conselho Nacional de Desenvolvimento Científico e Tecnológico) pelo apoio concedido no projeto Jovem Pesquisador no. 570316/2008, e à Empresa Esteio Engenharia de Aerolevantamentos S.A. pelo fornecimento da ortoimagem RGB.

\section{Referências bibliográficas}

BARSI A., HEIPKE C., WILLRICH F. 2002. Junction extraction by artificial neural network system jeans. In: ISPRS Technical Commission III Symposium 2002. Photogrammetry Comput Vision, PCV'02, Graz 2002 Internacional. Arch. Photogrammetry and Remote Sensing. Graz, Australia, 34,(b18), 9-13.

BAUMGARTNER A, ECKSTEIN W., HEIPKE C., HINZ S., MAYER H, RADIG B, STEGER C., WIEDEMANN C. 1999. T-rex: tum research on road extraction. In: Heipke, C., Mayer, H. (Eds.), Festschrift fur Prof. Dr. Ing. Heinrich Ebner zum 60. Geburtstag, Lehrstuhl fur Photogrammetrie und Fernerkundung, Technische Universitat Munchen, 43-64.

BAUMGARTNER A, STEGER C., MAYER C., ECKSTEIN W., BNER H. 1999. Automatic road extration based on multi-scale, grouping, and context. In: Photogrammetric Engineering and Remote Sensing, 65 (7), 777-785.

BAUMGARTNER A., STEGER C.T., WIEDEMANN C., MAYER H., ECKSTEIN W., EBNER H. 1996. Update of Roads in GIS from Aerial Imagery: Verification and Multi-Resolution Extraction. In: IAPRS International archieves of Photogrammetry and Remote Sensing, vol. XXXI, part. b3, 53-58.
DAL POZ A. P, AGOURIS P, GYFTAKIS S. 2000. Metodologias para a Extração Semi-automática de rodovias em imagens digitais: In: IV Congresso Brasileiro de Cadastro Técnico Multifinalitário, Floriánópolis - SC: UFSC.

DAL POZ A. P, AGOURIS P. 2000a. Extração semi-automática de rodovias usando programação dinâmica. In: II Workshop Brasileiro de Geoinformática, São Paulo, 3-9.

DAL POZ A. P, AGOURIS P. 2000b. Georeferenced road extration and formulation of hypoteheses for new road segments. In: Spie's 12th annual international symposium on aerospace/defense sensing, simulation, and control, V. 4054, 2000, Orlando-FI, Usa - Conference Automated Geo-Spatial Image and Data Exploitation, Orlano-FL, USA, 56-65.

DAL POZ A. P, AGOURIS, P. 2001. Um algoritmo de otimização global para a extração de rodovias em imagens digitais. In: Revista Brasileira de Cartografia, 53, 65-72.

DAL POZ A. P, GALLIS R.A., SILVA J.F.C. 2010. Extração semiautomática de rodovia baseada em um par estéreo de imagens aéreas e em otimização por programação dinâmica no espaço-objeto. In: Boletim de Ciências Geodésicas, sec. artigos, Curitiba, 16 (2), 189-209. 
DAL POZ A. P, GYFTAKIS S., AGOURIS P. 2000. Semi-automated Road extraction: Comparision of methodologics and experiments. In: DC 2000 ASPRS ANNUAL CONFERENCE, 2000. Washington-DC, EUA. D.

DAL POZ A. P, HABIB A.F., MARCATO V.J., CORREIA L.S. 2009. Uso de dados fotogramétricos no refinamento geométrico de contornos de telhados de edifícios extraídos de dados laser. In: Boletim de Ciências Geodésicas, sec. artigos, Curitiba, 15 (4), 594-614.

DAL POZ A. P, VALE G. M, OLIVEIRA, R.M.S. 2003. Extração semiautomática de rodovias em imagens digitais usando metodologias baseadas em programação dinâmica e contorno ativo. In: XXI Congresso Brasileiro de Cartografia, Belo Horizonte, Minas Gerais, 134-f14.

DAL POZ A. P, ZANIN R.B, VALE G.M. 2005. Extração automática da malha viária em imagens de média e alta resolução de cenas rurais. In: Anais XII Simpósio Brasileiro de Sensoriamento Remoto, Goiânia - GO, 16-21,.1183-1190.

DAL POZ A. P, ZANIN R.B., VALE G.M. 2007. Extração automática de feições rodoviárias em imagens digitais. In: SBA Controle \& Automação, 18 (1), 44-54.

DAL POZ A. P. 2002. Modelos e estratégias para a extração de malha viária em imagens digitais. Relatório FAPESP, Universidade Estadual Paulista (UNESP).

GAUTAMA S., GOEMAN W., D'HAEYER J. 2004. Robust detection of road junctions in VHR images using na improved ridge detector. In: International Archives of Photogrammetry, Remote Sensing and Spatial Information Sciences, 35 (b3), 815-819.

GERKE M., 2006. Automatic quality assessment of road databases using remotely sensed imagery. In: Wissenschaftliche Arbeiten der Fachrichtung Geodasie und Geoinformatik der Leibniz Universitat Hannover 261, and Deutsche Geodatische Kommission, Reihe C, n. 599. Beck Verlag Munchen Germany, p.105.

GÜLCH E. 2000. Digital systems for automated cartographic feature extraction. In: IAPRS - International archieves of Photogrammetry and Remote Sensing, vol. 32, part. b2, p.241256, 2000.

GWINNER K., HAUBER E., HOFFMANN H., SCHOLTEN F., JAUMANN R., NEUKUM G., PUGLISI G., COLTELLI M. 1999. The HRSC-A Experiment on High Resolution Multispectral Imaging and DEM Generation at the Aeolian Islands. In: 13th Int. Conf. on Applied Geologic Remote Sensing, Vancouver B.C., Canada, 1, 560-569.

HINZ S, BAUMGARTNER A., STEGER C., MAYER H., ECKSTERIN W., EBNER H., RADING B. 1999. Road Extraction in Rural and Urban Areas. In: Proc. of Semantic Modelling for the Acquisition of Topographic Information from Images and Maps (SMATI), Müchen. Ed. Förstner, Liedtke and Bückner.

HINZ S. 2008. Automatic object extraction for change detection and gis update. In: The International Archives of the Photogrammetry, Remote Sensing and Spatial Information Sciences. ISPR08. Vol. XXXVII, 277-284.

HOSOMURA T. 2008. Road extraction from high resolution satellite image by using circle area. In: The International Archives of the Photogrammetry, Remote Sensing and Spatial Information Sciences. ISPR08. Vol. XXXVII.

ISHIBASHI,R. 2008. Extração de segmentos de rodovias em imagens de resolução variadas usando o princípio de bordas paralelas. 136 f. Dissertação de Mestrado pela Faculdade de Ciências e Tecnologia - UNESP, Presidente Prudente - SP.

KUMAR K.V., MARTHA T.R., ROY P.S. 2006. Mapping damage in the Jammu and Kashmir caused by 8 October $2005 \mathrm{Mw} 7.3$ earthquake from the Cartosat- 1 and Resourcesat-1 imagery. In: International Journal of Remote Sensing, 27 (20), 44494459.

LIN X.G., ZHANG J.X., LIU Z. J., SHEN J. 2008. Semi-automatic extraction of ribbon roads form high resolution remotely sensed imagery by cooperation between angular texture signature and template matching. In: The International Archives of the Photogrammetry, Remote Sensing and Spatial Information Sciences. ISPR08. Vol. XXXVII.

MAKTAV D. 2009. Integration of remote sensing and SIG for archaeological investigations. In: International Journal of Remote Sensing, 30 (7), 1663-1673.

MAYER H., LAPTEV I., BAUMGARTNER, A. 1998. Multi-scale and snakes for automatic road extraction. In: 5th Europ. Conf. on Comput. Vision, 1, 720-733.

MAYUNGA S.D., COLEMAN D.J., ZHANG Y.A 2007. Semi-automated approach for extracting buildings from QuickBird imagery applied to informal settlement mapping. In: International Journal of Remote Sensing, 28 (10), 2343-2357.

MENA J.B. 2003. State of the art on automatic toad extraction for SIG updte: a novel classification. Pattern Recogniiton Lett. 24 (16), 3037-3058.

MIRNALINEE T.T., SUKHENDU DAS, VARGHESE K. 2009. Integration of Region and Edge-based information for Efficient Road Extraction from High Resolution Satellite Imagery. In: Advances in Pattern Recognition, 2009. ICAPR '09. Seventh International Conference, 373-376.

NAISSA B.L, SANTOS D.J., ANTUNES A.F.B. 2009. Segmentação de imagens e classificação baseada em regras de conhecimento como novas abordagens para o mapeamento do uso da terra no Estado do Paraná. In: Anais XIV Simpósio Brasileiro de Sensoriamento Remoto, Natal, Brasil, 25-30 abril, 989-996.

PATERAKI M.N., BALTSAVIAS E. 2002. Adaptive multi-image matching algorithm for the airborne digital sensor ADS40. In: Proceedings of the Map Asia 2002, Asian Conference on SIG, GPs, Aerial Photography and Remote Sensing, Bangkok, Thailand, 7-9 aug.

PATERAKI M.N., BALTSAVIAS, E.P. 2003. Analysis and performance of the adaptive multi-image matching algorithm for airborne digital sensor ADS40. In: Institute of Geodesy and Photogrammetry, EHTZ-Hoenggerberg, Zürich, Switzerland.

QUINLAN J.R. 1987. Simplifying decision trees. International Journal of Man-MachineStudies, 12, 221-234.

QUINLAN J.R. 1993. C4.5: Programs for Machine Learning. Morgan Kaufmann Publishers, San Mateo, USA.

QUINLAN J.R. 1994. C4.5: Programs of Machine Learning. Machine Learning, 16 Academic Publish, Manufacture in The Netherlands, Boston, 235-240.

QUINLAN J.R. 1996. Improved use of continuous attributes in C4.5. Journal of Artificial Intelligence Research 4, 77-90.

RAVANBAKHSH M., HEIPKE C., PAKZAD K. 2007. Road junction extraction from high resolution aerial images. In: Proceeding of International Archives of Photogrammetry, Remote Sensing and Spatial Information Sciences. Munich, Germany, 131-138.

SANTOS D.R., DALMOLIN Q., ARAKI H. 2006. Detecção automática de veículos com uso de imagens de Intensidade LiDAR e anaglifo. In: RBC - Revista Brasileira de Cartografia, 58 (3).

SARABANDI P., YAMAZAKI F., MATSUOKA M., KIREMIDJIAN A. 2004. Shadow detection and radiometric restoration in satellite high resolution images. In: Geoscience and Remote Sensing Symposium, 2004, IGARSS '04, Proceedings, 2004 IEEE International, Anchorage, Alaska, USA, 6, 3744-3747.

STEGER C. 1996. An unbiased detector of curvilinear structures, Technical Report FGBV-96-03, Forschungsgruppe 
Bildverstehen (FG BV), Informatik IX, Technische Universitat Munchen.

STEGER C., MAYER H., RADIG B. 1997. The Role of Grouping for Road Extraction. In: Automatic Extraction of Man-Made Objects from Aerial and Space Images (II), Armin Gruen, Emmanuel Baltsavias, Olof Henricsson (Editors), Birkhäuser Verlag, 245-256.

SHUKLA V., KANTH R.C., RAMACHANDRAN R. Semi-Automatic Road extraction algorithm for hig resolution images using path following approach, In: III Indian Conference on Computer Vision, Graphics and Imagem Processing, Ahmedabad - India.

TEOH C., SOWMYA A. 2000. Junction Extraction form high resolution images by compisite learning. In: International Archives of Photogrammetry and Remote Sensing, vol. XXXIII, $882-888$.

VALE G.M, DAL POZ A.P. 2003. Metodologia modificada de programação dinâmica para a extração acurada do eixo de rodovias em imagens digitais. In: Revista Brasileira de Cartografia, 55 (1), 11-20.

WEKA 3: Data Minig Software in Java. Disponível em: <http://www.cs.waikato. ac.nz/ml/weka>. Acesso em:24/06/2010.

WIEDEMANN C. 2002. Improvement of Road Crossing Extraction and External Evaluation of the Extraction Results. In: Proc. of the ISPRS Commision III Symposium on Photogrammetric Computer Vision (PCV'02), Graz/Austria 09.-13.09.2002. Institute for Computer Graphics and Vision, Graz University of Technology, vol. XXXIV, 297-300.

WITTEN I.H., FRANK E. 2005. Data mining: pratical machine learning tools and techiniques. 2 ed. San Francisco: Morgan Kaufmann Publishers.

ZANIN R.B. 2004. Metodologia automática para extração de cruzamento de rodovias em imagens de alta resolução. $121 \mathrm{f}$. Dissertação de Mestrado pela Faculdade de Ciências e Tecnologia - UNESP, Presidente Prudente - SP.

ZHANG C. 2004. Towards and operation system for automated updating for road database by integration of imagery and geodata. In: ISPRS Journal of Photogrammetry and Remote Sensing 58, 166-186.

ZHOU J., BISCHOF W.F., CAELLI, T. 2005. Robust and efficient road tracking in aerial images. In: Stilla $U$, Rottensteiner F, Hinz S. In: CMRT05. IAPRS International Archives of Photogrammetry and Remote Sensing, Vienna, Austria, 36. 